\title{
IMAGING DISEASES OF THE PEDIATRIC ESOPHAGUS
}

\author{
Mark C. LISZEWSKI \\ Montefiore Medical Center \\ and Albert Einstein College of Medicine \\ 111 East 210th Street, Bronx \\ NY 10467, USA \\ ${ }^{*}$ Corresponding author: \\ markcliszewski@gmail.com \\ Tel.: + 718-920-7748 \\ Fax.: + 718-798-7983
}

Received /Accepted: July 4, 2015

Key words: Pediatric esophagus - Esophagram - Congenital esophageal disorders.

Numerous conditions can affect the pediatric esophagus and it is essential that physicians caring for children are familiar with the radiographic evaluation of esophageal disorders. Diseases include congenital conditions such as esophageal atresia, tracheoesophageal fistula, congenital esophageal stenosis, foregut duplication cyst, esophageal bronchus, vascular rings, and pulmonary sling, inflammatory conditions such as eosinophilic esophagitis and epidermolysis bullosa, traumatic conditions such as ingested foreign body and iatrogenic esophageal injury, neoplasms such as leiomyoma, carcinoma, and lymphoma, infectious conditions such as herpes simplex virus, cytomegalovirus and Candida esophagitis, gastroesophageal reflux and achalasia (1). The radiographic assessment of these conditions typically begins with an esophogram, which utilizes fluoroscopy and an ingested contrast agent.

In the most recent issue of Peadiatrics Today, Ključevšek (2) provide a review of the pediatric esophogram and offer a useful over- view for pediatricians, radiologists and other clinicians caring for children with diseases of the esophagus. After a review of the normal anatomy and physiology of the esophagus, the authors describe the technique and imaging findings in a normal esophogram focusing on the oral, pharyngeal and esophageal phases. They then discuss the appearance of several esophageal diseases on esophogram including disordered esophageal motility, congenital conditions, esophageal stenosis, diseases of the gastroesophageal junction (gastroesophageal reflux, achalasia, and hiatal hernia), outside impressions on the esophagus from vascular rings and pulmonary sling and foreign bodies. This pictorial essay provides a useful overview of the broad range of disease processes that can affect the esophagus and their appearance on esophogram.

In many diseases of the pediatric esophagus an esophogram is sufficient to provide all the diagnostic information that is needed, but in some instances additional imaging is required. For example, when an esophogram finds an abnormal outside impression on the esophagus, cross section imaging such as CT or MRI is often required to further characterize the abnormality. In these cases cross sectional imaging may find a duplication cyst, vascular ring, pulmonary sling or mediastinal mass $(1,3,4)$. In cases of vascular ring and sling CT angiogram (CTA) or MR angiogram (MRA) is often extremely useful in defining the vascular anomaly, evaluating esophageal compression, and identifying compression of the airway which is often also 
present. Both CTA and MRA provide excellent definition of vascular structures and both can produce 3 dimensional reformations which are often very useful in defining the vessels' relationship to the esophagus, airway, and other mediastinal structures. MRA is a particularly attractive modality because it uses no ionizing radiation, and some MRA techniques can be performed without contrast injection. However, young patients often require sedation for MRA, which carries risk. CTA does involve the use of ionizing radiation and intravenous contrast, but sedation can often be avoided and CT provides more detailed evaluation of the airways than MRI (4). Therefore, when choosing between CTA and MRA the advantages and disadvantages of each modality must be assessed in each individual case.

In summary, esophageal abnormalities are frequent in children and imaging plays a key role in their diagnosis. Esophogram is the first line imaging test and in most cases is sufficient to provide all the diagnostic information that is needed. A minority of patients will require cross sectional imaging. It is essential that physicians caring for children are familiar with the radiographic evaluation of esophageal disorders and Ključevšek (2) provide a useful overview of the pediatric esophogram.

Conflict of interest: The author declares that he has no conflict of interest.

\section{References}

1. Hryhorczuk AL, Lee EY, Eisenberg RL. Esophageal abnormalities in pediatric patients. AJR Am J Roentgenol. 2013; 201(4):W519-32.

2. Ključevšek D. A pictorial essay on radiography of swallowing and esophageal disorders. Paediatrics Today. 2015;11(1):10-23.

3. Callahan MJ, Taylor GA. CT of the pediatric esophagus. AJR Am J Roentgenol. 2003;181(5):1391-6.

4. Kellenberger CJ. Aortic arch malformations. Pediatr Radiol. 2010;40(6):876-84. 